\title{
Perfect codes in Cartesian products of 2-paths and infinite paths
}

\author{
Paul Dorbec*, Michel Mollard ${ }^{\dagger}$ \\ ERTé Maths à Modeler, Groupe de recherche GéoD, \\ Laboratoire Leibniz, \\ 46 av. Félix Viallet, 38031 Grenoble CEDEX, FRANCE
}

Submitted: Feb 24, 2005; Accepted: Nov 22, 2005; Published: Nov 29, 2005

Mathematics Subject Classifications: 05C69,05C70,94B60

\begin{abstract}
We introduce and study a common generalization of 1-error binary perfect codes and perfect single error correcting codes in Lee metric, namely perfect codes on products of paths of length 2 and of infinite length. Both existence and nonexistence results are given.
\end{abstract}

\section{Introduction}

Perfect codes appeared in error correcting codes theory during the late 40's with the work of Golay and Hamming [3, 6]. They constructed perfect binary single-error correcting codes of length $n$ where $n=2^{q}-1$ for some integer $q$.

Later, Golomb and Welch [4,5] proved, for any length $n$, the existence of perfect single-error correcting codes in Lee metric. Such codes can be considered either as regular periodic tilings of the euclidean space $\mathbb{R}^{n}$ by Lee spheres of radius 1 or as periodic tilings of the grid $\mathbb{Z}^{n}$ by balls of radius 1 . Perfect codes have also been studied on other alphabets or mixed one (see [2]).

In [1], Biggs introduced perfect codes in graphs. From this point of view, a perfect binary single-error correcting code of length $k$ is a perfect code on the hypercube $Q_{k}$, and a tiling of the grid $\mathbb{Z}^{n}$ by balls of radius 1 is nothing more than a perfect code of $\mathbb{Z}^{n}$.

*ENS Lyon, paul.dorbec@imag.fr

${ }^{\dagger}$ CNRS, michel.mollard@imag.fr

¥This work was partially supported by PROTEUS 
Both $Q_{k}$ and the grid $\mathbb{Z}^{n}$ are cartesian products of paths either of length $2\left(P_{2}\right)$ or of infinite length $\left(P_{\infty}\right)$. We study the existence of perfect codes on the mixed product $P_{\infty}^{n} \square P_{2}^{k}=\mathbb{Z}^{n} \square Q_{k}$.

In the next section, we give the definitions we will use along this paper. Section 3 presents classical results on error correcting codes. In section 4, we construct new codes, and in section 5 , we prove some inexistence results. The last section summarizes what we know and what is still open.

\section{Definitions}

Given $G_{1}=\left(V_{1}, E_{1}\right)$ and $G_{2}=\left(V_{2}, E_{2}\right)$ two graphs, the cartesian product $G_{1} \square G_{2}$ is the graph with vertex set $V_{1} \times V_{2}$ and satisfying $\left(x_{1}, x_{2}\right)\left(y_{1}, y_{2}\right) \in E\left(G_{1} \square G_{2}\right)$ if and only if $x_{1} y_{1} \in E_{1}$ and $x_{2}=y_{2}$ or $x_{2} y_{2} \in E_{2}$ and $x_{1}=y_{1}$. We will use the notation $G^{n}$ for the graph $G \square G \square \ldots \square G$ ( $n$ times).

The hypercube of dimension $k$ is the graph $Q_{k}$ whose vertices are the words of length $k$ over the alphabet $\{0,1\}$, and where two vertices are adjacent if they differ in exactly one place. Notice that $Q_{1}$ is $P_{2}$ the path with 2 vertices and that $Q_{k+1}=Q_{k} \square P_{2}$. For two vertices $u$ and $v$ in $Q_{k}$, we will denote by $u+v$ the vertex $\left(\left(u_{1}+v_{1}\right),\left(u_{2}+v_{2}\right), \ldots,\left(u_{k}+v_{k}\right)\right)$ where + is the sum modulo 2 .

The infinite grid $\mathbb{Z}^{n}$ is the graph whose vertices are the words of length $n$ over the alphabet $\mathbb{Z}$ and where two vertices are adjacent if and only if they differ by 1 in exactly one place. Notice that if we denote by $P_{\infty}$ the two ways infinite path, we have also $\mathbb{Z}^{1}=P_{\infty}$ and $\mathbb{Z}^{n+1}=\mathbb{Z}^{n} \square P_{\infty}$.

We will consider in the same idea the vertices of the cycle of length $l$, denoted by $C_{l}$, as the elements of $\mathbb{Z} / l \mathbb{Z}$. For two vertices $u$ and $v$, we will denote by $u+v$ the vertex $(u+v) \bmod l$.

From now on, we will work on the cartesian product $\mathbb{Z}^{n} \square Q_{k}$. This graph can also be considered as follows : the vertices are words of length $n+k$ whose $n$ first symbols are in $\mathbb{Z}$ and the $k$ others are in $\{0,1\}$. Two vertices are adjacent if they differ by 1 in exactly one place. Notice that this graph is regular with degree $2 n+k$. Moreover, $\mathbb{Z}^{n} \square Q_{0}=\mathbb{Z}^{n}$ and $\mathbb{Z}^{0} \square Q_{k}=Q_{k}$.

For two vertices $x$ and $y$, we will denote by $d(x, y)$ the classical distance on graphs. For our graph $\mathbb{Z}^{n} \square Q_{k}$,

$$
d\left(\left(x_{1}, x_{2}, \ldots, x_{n+k}\right),\left(y_{1}, y_{2}, \ldots, y_{n+k}\right)\right)=\sum_{i=1}^{n+k}\left|x_{i}-y_{i}\right|
$$

If $n=0$, this is the Hamming distance, and if $k=0$, this is the Manhattan distance.

For an integer $r$ and a vertex $c$, we call ball of radius $r$ centered on $c$ the set of vertices $v$ such that $d(c, v) \leq r$. In this paper, we will only consider balls of radius 1 .

In a graph, a single error correcting code (or code for shorter) is a set of vertices such that any two code's vertices are at distance at least 3. This is equivalent to say that the balls centered on these vertices are disjoint. 


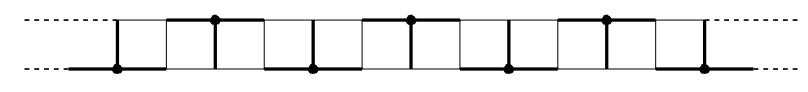

Figure 1: A perfect code on $\mathbb{Z}^{1} \square Q_{1}$

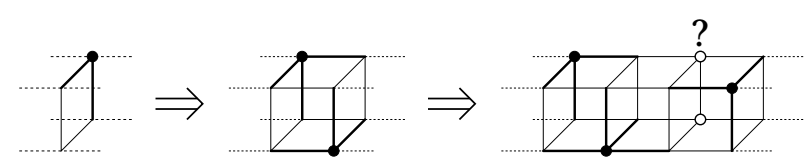

Figure 2: There exists no perfect code on $\mathbb{Z}^{1} \square Q_{2}$

A code is said to be perfect if any vertex of the graph is at distance at most one of a code's vertex. It also means that any vertex belongs to a ball centered on a code's vertex and thus that these balls form a tiling of the graph.

Figure 1 shows a perfect code in $\mathbb{Z}^{1} \square Q_{1}$. But a perfect code does not always exists as figure 2 shows.

Let $G$ be a finite graph. On $\mathbb{Z}^{n} \square G$, we will say that a code is $i$-periodic $(i \in\{1, \ldots, n\})$ if there exists a positive integer $p_{i}$ such that for any vertex $x=\left(x_{1}, x_{2}, \ldots, x_{n}, v\right)$ (where $\left.\forall i, x_{i} \in \mathbb{Z}, v \in V(G)\right)$, the vertex $\left(x_{1}, x_{2}, \ldots, x_{i-1},\left(x_{i}+p_{i}\right), x_{i+1}, \ldots, x_{n}, v\right)$ is in the code if and only if $x$ is in the code. $p_{i}$ is called the $i$-period. A code is periodic of period $\left(p_{1}, p_{2}, \ldots, p_{n}\right)$ if it is $i$-periodic of $i$-period $p_{i}$ for all $i$.

Proposition 1 Let $S$ be a periodic code on $\mathbb{Z}^{n} \square G$ of period $\left(p_{1}, p_{2}, \ldots, p_{n}\right)$. There exists some set $T$ of words $t=\left(t_{1}, t_{2}, \ldots, t_{n}, v\right)$ with $\forall i \in\{1 \ldots, n\}, 0 \leq t_{i}<p_{i}$ and $v \in V(G)$ such that $S$ is the set of words

$$
\left\{\left(t_{1}+\alpha_{1} p_{1}, t_{2}+\alpha_{2} p_{2}, \ldots, t_{n}+\alpha_{n} p_{n}, v\right) \mid t \in T, \alpha_{i} \in \mathbb{Z}\right\}
$$

\section{Perfect codes deduced from known constructions}

From the classical results on error correcting codes, we get:

Theorem 2 If $n=0$, there exists a perfect code on $\mathbb{Z}^{n} \square Q_{k}$ if and only if there exists an integer $p$ such that $k=2^{p}-1$.

Examples of these perfect codes are the classical Hamming codes [6], but when $k \geq 15$, other perfect codes with the same length are known (for a survey on this topic, see [2]).

Theorem 3 There exists a i-periodic perfect code on $\mathbb{Z}^{n} \square G$ of $i$-period $p_{i}$ if and only if a perfect code on the graph $\mathbb{Z}^{n-1} \square C_{p_{i}} \square G$ exists.

Proof : Let $S$ be a perfect code on $\mathbb{Z}^{n-1} \square C_{p_{i}} \square G$. Then one can easily check that the set of words

$$
\left\{\left(x_{1}, x_{2}, \ldots, x_{n-1}, c+\alpha p_{i}, v\right) \mid\left(x_{1}, x_{2}, \ldots, x_{n-1}, c, v\right) \in S, \alpha \in \mathbb{Z}\right\}
$$



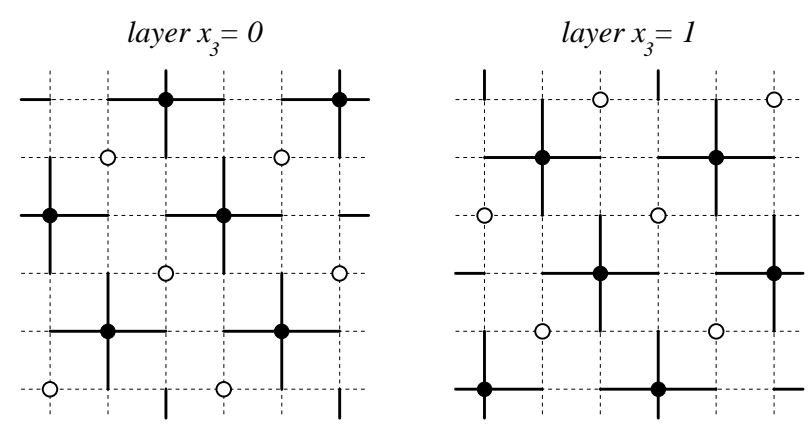

Figure 3: A perfect code on $\mathbb{Z}^{2} \square Q_{1}$

is a perfect code of $\mathbb{Z}^{n} \square G$.

On the other hand, let us consider a perfect $i$-periodic code $S$ on $\mathbb{Z}^{n} \square G$ of $i$-period $p_{i}$. Without loss of generality, we can suppose that $i=n$. The set of words

$$
\left\{\left(x_{1}, x_{2}, \ldots, x_{n}, v\right) \mid\left(x_{1}, x_{2}, \ldots, x_{n}, v\right) \in S, 0 \leq x_{n}<p_{n}\right\}
$$

is a perfect code on $\mathbb{Z}^{n-1} \square C_{p_{n}} \square G$.

Thus, there exists a periodic perfect code on the grid $\mathbb{Z}^{n}$ of period $\left(p_{1}, \ldots, p_{n}\right)$ if and only if a perfect code on the graph $C_{p_{1}} \square \ldots \square C_{p_{n}}$ exists.

In 1968, Golomb and Welch [4] proved the existence in Lee metric of perfect one error correcting codes for any word length $n$ over an alphabet of $2 n+1$ elements. This is a perfect code on the graph $C_{2 n+1}^{n}$, and this implies the following well known result.

Theorem 4 If $k=0$, for any $n \in \mathbb{N}$, there exists a periodic perfect code on $\mathbb{Z}^{n} \square Q_{k}$.

\section{Constructing perfect codes}

A perfect code on $\mathbb{Z}^{2} \square Q_{1}$ exists (see figure 3 ). We can consider this construction as a tiling of $\mathbb{Z}^{2}$ with balls of radius 1 and of radius 0 . The set of balls of radius 1 is obtain from the set of balls of radius 0 by a translation. With this approach, such a tiling can be generalized for any $n$ on $\mathbb{Z}^{n} \square Q_{1}$.

But it is a special case of a more general construction we present hereby.

Theorem 5 There exists a perfect code on $\mathbb{Z}^{n} \square Q_{k}$ whenever there are some $\alpha, \beta \in \mathbb{N}^{*}$ such that $k=2^{\alpha}-1$ and $n=\beta 2^{\alpha-1}$.

Proof : Let $k=2^{\alpha}-1$ and $n=\beta 2^{\alpha-1}$. We will construct a perfect code on $\mathbb{Z}^{n} \square Q_{k}$.

From theorem 2, there exists a perfect code $A_{0}$ on $Q_{k}$. We denote by $e_{1}, \ldots, e_{k}$ the $k$ vertices of $Q_{k}$ at distance 1 of $(0, \ldots, 0)$ and by $A_{i}$ the set $\left\{c+e_{i} \mid c \in A_{0}\right\}$. By definition of a perfect code, any $A_{i}$ is also a perfect code on $Q_{k}$ and $A_{0}, A_{1}, \ldots, A_{k}$ is a partition of $V\left(Q_{k}\right)$. To prove the theorem, we will construct a mapping from $\mathbb{Z}^{n}$ that characterizes a perfect code on $\mathbb{Z}^{n} \square Q_{k}$. Denote by $x=\left(x_{1}, x_{2}, \ldots, x_{n}\right)$ an element of $\mathbb{Z}^{n}$. 


\section{THE CASE $\beta$ EVEN:}

Let $\beta=2 p$. We have $n=p(k+1)$.

We define the following mapping from $\mathbb{Z}^{p(k+1)}$ to $\mathbb{Z} /(k+1)(2 p+1) \mathbb{Z}$ :

$$
\begin{aligned}
f(x)= & \sum_{i=0}^{k} \sum_{j=1}^{p}((2 p+1) i+j) x_{i p+j} \bmod (k+1)(2 p+1) \\
\text { That is } f(x)= & x_{1}+2 x_{2}+\ldots+p x_{p} \\
& +(2 p+2) x_{p+1}+(2 p+3) x_{p+2}+\ldots+(3 p+1) x_{2 p} \\
& +\ldots \\
& +(k(2 p+1)+1) x_{k p+1}+\ldots+(k(2 p+1)+p) x_{(k+1) p} \\
& \bmod (k+1)(2 p+1)
\end{aligned}
$$

We claim that the set $C=\left\{(x, v) \mid \exists i \in\{0, \ldots, k\}\right.$ with $\left.f(x)=i(2 p+1), v \in A_{i}\right\}$ is a perfect code of $\mathbb{Z}^{n} \square Q_{k}$. To prove this, we will use the following lemma.

Lemma 6 For any $x \in \mathbb{Z}^{n}$ and $\theta \in\{1, \ldots,(k+1)(2 p+1)-1\}$, there exists exactly one neighbour $y$ of $x$ such that $f(y)=f(x)+\theta$ if $\theta \neq 0 \bmod 2 p+1$ and no neighbour if $\theta=0 \bmod 2 p+1$.

Proof : Let $\theta \in\{1, \ldots,(k+1)(2 p+1)-1\}$ satisfy $\theta \neq 0 \bmod 2 p+1$. There exist $i \in\{0, \ldots, k\}, j \in\{1, \ldots, 2 p\}$ such that $\theta=(2 p+1) i+j$. If $j \leq p$ then $f\left(x_{1}, \ldots, x_{i p+j}+\right.$ $\left.1, \ldots, x_{n}\right)=f(x)+\theta$. Else, $f\left(x_{1}, \ldots, x_{(k-i) p+(2 p+1-j)}-1, \ldots, x_{n}\right)=f(x)+\theta$ (notice that $k-i \in\{0, \ldots, k\}$ and $2 p+1-j \in\{1, \ldots, p\})$. We have thus considered $(k+1) 2 p=2 n$ distinct neighbours of $x$, so every neighbour of $x$. Therefore there are no neighbour left for the case $\theta=0 \bmod 2 p+1$.

Suppose that $C$ is not a code with minimum distance 3 . Then there exist two distinct vertices $(x, v)$ and $\left(x^{\prime}, v^{\prime}\right) \in C$ at distance less or equal to 2 . Let $i$ and $i^{\prime}$ be the integers such that $f(x)=i(2 p+1)$ and $f\left(x^{\prime}\right)=i^{\prime}(2 p+1)$. We have $v \in A_{i}$ and $v^{\prime} \in A_{i^{\prime}}$.

$1^{s t}$ case : $x=x^{\prime}$. Thus $v$ and $v^{\prime}$ are in a same $A_{i}$ and $v \neq v^{\prime}$ so $d\left(v, v^{\prime}\right) \geq 3$ : a contradiction.

$2^{\text {nd }}$ case $: d\left(x, x^{\prime}\right)=1$. From lemma $6, f(x)-f\left(x^{\prime}\right) \neq 0 \bmod 2 p+1$ but $f(x)-f\left(x^{\prime}\right)=$ $\left(i-i^{\prime}\right)(2 p+1):$ a contradiction.

$3^{r d}$ case : $d\left(x, x^{\prime}\right)=2$. Then $v=v^{\prime}$; and since $A_{0}, \ldots, A_{k}$ is a partition of $V\left(Q_{k}\right)$, $i=i^{\prime}$ and $f(x)=f\left(x^{\prime}\right)$. Let $u$ be a common neighbour of $x$ and $x^{\prime}$. We have $f(u)-f(x)=f(u)-f\left(x^{\prime}\right)$ which is impossible by lemma 6 .

We only have to prove now that this code is perfect. Let $(x, v)$ be a vertex of $\mathbb{Z}^{n} \square Q_{k}$. If $f(x)=i(2 p+1)$ then $A_{i}$ being a perfect code, there exists $v^{\prime} \in A_{i}\left(\right.$ so $\left.\left(x, v^{\prime}\right) \in C\right)$ such that $d\left((x, v),\left(x, v^{\prime}\right)\right) \leq 1$. Else, since $A_{0}, \ldots, A_{k}$ is a partition of $V\left(Q_{k}\right)$, there exists $i \in\{0, \ldots, k\}$ such that $v \in A_{i}$. By lemma 6 , there exists a neighbour $x^{\prime}$ of $x$ such that $f\left(x^{\prime}\right)=i(2 p+1)$. Thus, $\left(x^{\prime}, v\right) \in C$. 


\section{THE CASE $\beta$ ODD:}

Let $\beta=2 p+1$. Notice that $\frac{k+1}{2}$ is an integer. We have $n=(2 p+1) \frac{k+1}{2}$.

We define the following mapping from $\mathbb{Z}^{n}$ to $\mathbb{Z} /(k+1)(2 p+2) \mathbb{Z}$ :

$$
\begin{aligned}
g(x)= & \sum_{i=0}^{k} \sum_{j=1}^{p}((2 p+2) i+j) x_{i p+j} \\
& +\sum_{l=1}^{\frac{k+1}{2}}(p+1)(2 l-1) x_{(k+1) p+l} \bmod (k+1)(2 p+2) \\
\text { That is } g(x)= & x_{1}+2 x_{2}+\ldots+p x_{p} \\
& +(2 p+3) x_{p+1}+(2 p+4) x_{p+2}+\ldots+(3 p+2) x_{2 p} \\
& +\ldots \\
& +(k(2 p+2)+1) x_{k p+1}+\ldots+(k(2 p+2)+p) x_{(k+1) p} \\
& +(p+1) x_{(k+1) p+1}+\ldots+k(p+1) x_{(k+1) p+\frac{k+1}{2}} \\
& \bmod (k+1)(2 p+2)
\end{aligned}
$$

We claim that the set $C=\left\{(x, v) \mid \exists i \in\{0, \ldots, k\}\right.$ with $\left.g(x)=i(2 p+2), v \in A_{i}\right\}$ is a perfect code of $\mathbb{Z}^{n} \square Q_{k}$. To prove this, we will use the following lemma.

Lemma 7 For any $x \in \mathbb{Z}^{n}$ and $\theta \in\{1, \ldots,(k+1)(2 p+2)-1\}$, there exists exactly one neighbour $y$ of $x$ such that $g(y)=g(x)+\theta$ if $\theta \neq 0 \bmod 2 p+2$ and no neighbour if $\theta=0 \bmod 2 p+2$.

Proof : Let $\theta \in\{1, \ldots,(k+1)(2 p+2)-1\}$ satisfy $\theta \neq 0 \bmod 2 p+2$. There exist $i \in\{0, \ldots, k\}, j \in\{1, \ldots, 2 p+1\}$ such that $\theta=(2 p+2) i+j$.

- If $j<p+1$ then $g\left(x_{1}, \ldots, x_{i p+j}+1, \ldots, x_{n}\right)=g(x)+\theta$.

- If $j>p+1$ then $g\left(x_{1}, \ldots, x_{(k-i) p+(2 p+2-j)}-1, \ldots, x_{n}\right)=g(x)+\theta$ (notice that $k-i \in\{0, \ldots, k\}$ and $2 p+2-j \in\{1, \ldots, p\})$.

- If $j=p+1$ then $\theta=(p+1)(2 i+1)$ and

- if $i<\frac{k+1}{2}, g\left(x_{1}, x_{2}, \ldots, x_{(k+1) p+i+1}+1, \ldots, x_{n}\right)=g(x)+\theta$

- if $i \geq \frac{k+1}{2}, g\left(x_{1}, x_{2}, \ldots, x_{(k+1) p+k-i+1}-1, \ldots, x_{n}\right)=g(x)+\theta$ (notice that $k-$ $\left.i+1 \in\left\{1, \ldots, \frac{k+1}{2}\right\}\right)$.

We have thus considered $(k+1)(2 p+1)=2 n$ distinct neighbours of $x$, so every neighbour of $x$. Therefore, there are no neighbour left for the case $\theta=0 \bmod 2 p+2$.

Suppose that $C$ is not a code with minimum distance 3 . Then there exist two distinct vertices $(x, v)$ and $\left(x^{\prime}, v^{\prime}\right) \in C$ at distance less or equal to 2 . Let $i$ and $i^{\prime}$ be the integers such that $g(x)=i(2 p+2)$ and $g\left(x^{\prime}\right)=i^{\prime}(2 p+2)$. We have $v \in A_{i}$ and $v^{\prime} \in A_{i^{\prime}}$.

$1^{\text {st }}$ case : $x=x^{\prime}$. Thus $v$ and $v^{\prime}$ are in a same $A_{i}$ and $v \neq v^{\prime}$ so $d\left(v, v^{\prime}\right) \geq 3$ : a contradiction.

$2^{\text {nd }}$ case $: d\left(x, x^{\prime}\right)=1$. From lemma $7, g(x)-g\left(x^{\prime}\right) \neq 0 \bmod 2 p+2$ but $g(x)-g\left(x^{\prime}\right)=$ $\left(i-i^{\prime}\right)(2 p+2):$ a contradiction. 
$3^{\text {rd }}$ case : $d\left(x, x^{\prime}\right)=2$. Then $v=v^{\prime}$; and since $A_{0}, \ldots, A_{k}$ is a partition of $V\left(Q_{k}\right)$, $i=i^{\prime}$ and $g(x)=g\left(x^{\prime}\right)$. Let $u$ be a common neighbour of $x$ and $x^{\prime}$. We have $g(u)-g(x)=g(u)-g\left(x^{\prime}\right)$ which is impossible by lemma 7 .

We only have to prove now that this code is perfect. Let $(x, v)$ be a vertex of $\mathbb{Z}^{n} \square Q_{k}$. If $g(x)=i(2 p+2)$ then $A_{i}$ being a perfect code, there exists $v^{\prime} \in A_{i}\left(\right.$ so $\left.\left(x, v^{\prime}\right) \in C\right)$ such that $d\left((x, v),\left(x, v^{\prime}\right)\right) \leq 1$. Else, since $A_{0}, \ldots, A_{k}$ is a partition of $V\left(Q_{k}\right)$, there exists $i \in\{0, \ldots, k\}$ such that $v \in A_{i}$. By lemma 7, there exists a neighbour $x^{\prime}$ of $x$ such that $g\left(x^{\prime}\right)=i(2 p+2)$. Thus, $\left(x^{\prime}, v\right) \in C$.

Proposition 8 There exists a i-periodic perfect code on $\mathbb{Z}^{n+1} \square Q_{k}$ of $i$-period 4 if and only if a perfect code on the graph $\mathbb{Z}^{n} \square Q_{k+2}$ exists.

Proof : This proposition is an immediate consequence of theorem 3 since $Q_{2}=C_{4}$.

Corollary 9 If there exists an integer $p$ such that $2 n+k=2^{p}-1$, then their exists a perfect code on $\mathbb{Z}^{n} \square Q_{k}$.

Proof : This is a consequence of proposition 8 and theorem 2.

Corollary 10 There exists a perfect code on $\mathbb{Z}^{n} \square Q_{k}$ whenever there are some $\alpha, \beta, \gamma \in \mathbb{N}$ such that $k=2^{\alpha}-2 \gamma-1$ and $n=\beta 2^{\alpha-1}+\gamma$.

Proof : This is a consequence of theorem 5, proposition 8, and corollary 9.

\section{$5 \quad$ Nonexistence of perfect codes}

Theorem 11 Suppose that $k \geq 2 n$. Then there exists a perfect code on $\mathbb{Z}^{n} \square Q_{k}$ if and only if there exists an integer $p$ such that $2 n+k=2^{p}-1$.

Proof : From corollary 9, we know that a perfect code exists when $n$ and $k$ satisfy the condition.

For $x=\left(x_{1}, \ldots, x_{n}\right) \in \mathbb{Z}^{n}$, let $Q_{k}(x)$ be the set of vertices $\left(u_{1}, \ldots, u_{n+k}\right)$ of $\mathbb{Z}^{n} \square Q_{k}$ such that $u_{i}=x_{i}$ for any $1 \leq i \leq n$. Suppose there exists a perfect code $C$ on $\mathbb{Z}^{n} \square Q_{k}$.

Since $C$ is a perfect code, any vertex has to be in exactly one ball centered on a vertex of $C$. A ball centered on a vertex in $Q_{k}(x)$ contains $k+1$ vertices in $Q_{k}(x)$. A ball centered on some vertex in a $Q_{k}(y)$ such that $d(y, x)=1$ contains exactly one vertex in $Q_{k}(x)$.

We consider $\gamma$ the minimum number of vertices of the code we can find in a $Q_{k}(x)$. Let $x \in \mathbb{Z}^{n}$ satisfy $\left|Q_{k}(x) \cap C\right|=\gamma$. Let $\left(y_{i}\right)_{1 \leq i \leq 2 n}$ be the vertices of $\mathbb{Z}^{n}$ at distance 1 from $x$. We define $a_{i}=\left|Q_{k}\left(y_{i}\right) \cap C\right|-\gamma$. Let $a=\max \left(a_{i}\right)$ and $y$ be a $y_{i}$ such that $a_{i}=a$.

Suppose $a=0$, and thus that every $a_{i}$ is null. We consider the vertices of $Q_{k}(x)$. $(k+1) \gamma$ of them are in balls centered on vertices of $Q_{k}(x)$, and $2 n \gamma$ in balls centered on some vertex of $Q_{k}\left(y_{i}\right)$ for some $i$. Thus we have $(k+1) \gamma+2 n \gamma=2^{k}$. So $k+1+2 n$ is a factor of $2^{k}$, and there is some $p$ such that $2 n+k=2^{p}-1$. 


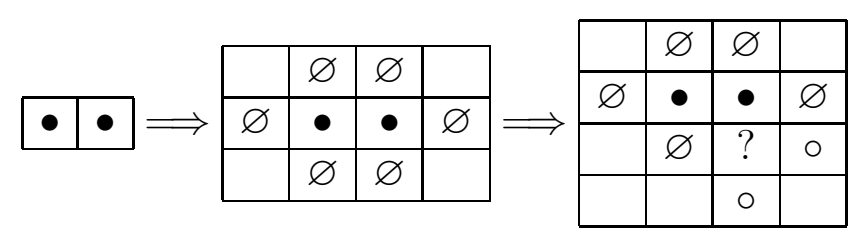

Figure 4: Nonexistence of a perfect code on $\mathbb{Z}^{2} \square Q_{2}$

Now suppose $a>0$. Counting the vertices of $Q_{k}(x)$, we get :

$$
(k+1) \gamma+\sum_{i=1}^{2 n}\left(a_{i}+\gamma\right)=2^{k}
$$

and by counting those of $Q_{k}(y)$ :

$$
(k+1)(\gamma+a)+\sum_{i=1}^{2 n}\left(\gamma+b_{i}\right)=2^{k}
$$

where $b_{i}$ are nonnegative integers defined in a way similar to the $a_{i}$ 's. Doing (2) - (1), we obtain $a(k+1)+\sum_{i=1}^{2 n} b_{i}=\sum_{i=1}^{2 n} a_{i}$. Then, since $\sum_{i=1}^{2 n} b_{i} \geq 0$ and $\sum_{i=1}^{2 n} a_{i} \leq 2 n a$, we have $a(k+1) \leq 2 n a$ and so $k<2 n$.

Proposition 12 There exist no perfect code on $\mathbb{Z}^{2} \square Q_{2}$ nor on $\mathbb{Z}^{3} \square Q_{2}$.

Proof : Notice that any $Q_{2}(x)$ may contain 1 vertex of the code $\left(Q_{2}\right.$ of type ' $\bullet$ ') or no vertex (type ' $\varnothing$ '). A $Q_{2}$ of type ' $\bullet$ ' has exactly 1 neighbour of type '•' while a type ' $\varnothing$ ' has exactly 4 neighbours of type ' $\bullet$ '. See figure 4 for $\mathbb{Z}^{2} \square Q_{2}$. A similar but tedious case analysis proves the nonexistence of a perfect code on $\mathbb{Z}^{3} \square Q_{2}$.

\section{Conclusion and open problems}

We recapitulate our results in table 1 where $\sqrt{ }$ means existence:

$a$ by theorem 2

$b$ by theorem 4

$c$ by theorem 2 and proposition 8

$d$ by theorem 5

$e$ by theorem 5 and proposition 8

and - means nonexistence by theorem 11 except for $-_{f}$ that are proven by proposition 12 .

Clearly, any empty case fulfilling would be interesting. Furthermore, this table suggest that there are no perfect code on $\mathbb{Z}^{n} \square Q_{k}$ when $k$ is even. By proposition 8 , it would be sufficient to prove this nonexistence when $k=2$. 


\begin{tabular}{|c|c|c|c|c|c|c|c|c|c|c|c|c|c|}
\hline$n \backslash k$ & 0 & 1 & 2 & 3 & 4 & 5 & 6 & 7 & 8 & 9 & 10 & 11 & \\
\hline 0 & $\sqrt{ }$ & $\sqrt{ } a$ & - & $\sqrt{a}$ & - & - & - & $\overline{\sqrt{ } a}$ & - & - & - & - & \\
\hline 1 & $\sqrt{b}$ & $\sqrt{ }_{c, d}$ & - & - & - & $\sqrt{c}$ & - & - & - & - & - & - & . \\
\hline 2 & $\sqrt{b}$ & $\sqrt{d}$ & $-f$ & $\sqrt{ }_{c, d}$ & - & - & - & - & - & - & - & $\sqrt{c}$ & \\
\hline 3 & $\sqrt{b}$ & $\sqrt{ }_{c, d}$ & $-f$ & & & & - & - & - & $\sqrt{ } c$ & - & - & . \\
\hline 4 & $\overline{\sqrt{b}}$ & $\sqrt{d}$ & & $\sqrt{d}$ & & & & $\sqrt{ }_{c, d}$ & - & - & - & - & \\
\hline 5 & $\sqrt{b}$ & $\sqrt{ }_{c, d}$ & & & & $\sqrt{c}$ & & & & & - & - & $\ldots$ \\
\hline 6 & $\sqrt{b}$ & $\sqrt{d}$ & & $\sqrt{ }_{c, d}$ & & & & & & & & & $\ldots$ \\
\hline 7 & $\sqrt{b}$ & $\sqrt{ }_{c, d}$ & & & & & & & & & & & $\cdots$ \\
\hline 8 & $\sqrt{b}$ & $\sqrt{d}$ & & $\sqrt{d}$ & & & & $\sqrt{d}$ & & & & & $\ldots$ \\
\hline 9 & $\sqrt{b}$ & $\sqrt{ }_{c, d}$ & & & & $\sqrt{e}$ & & & & & & & \\
\hline
\end{tabular}

Table 1: Existence of perfect codes on $\mathbb{Z}^{n} \square Q_{k}$

\section{References}

[1] N. Biggs, [1973] : "Perfect codes in graphs", J. Combin. Theory Ser. B 15, pp 289-296.

[2] G. Cohen, I. Honkala, S. Litsyn, A. Lobstein, [1997] : "Covering Codes", Chap 11 Elsevier.

[3] M. J. E. Golay, [1949] : "Notes on digital coding", Proc. IEEE 37, p 657.

[4] S.W. Golomb and L.R. Welch, [1968] : "Algebraic coding and the Lee metric", Proc. Sympos. Math. Res. Center, Madison, Wis., pp 175-194, John Wiley, New York.

[5] S.W. Golomb and L.R. Welch, [1970] : "Perfect codes in the Lee metric and the packing of polyominoes", SIAM J. Appl. Math. 18, pp 302-317.

[6] R. W. Hamming, [1950] : "Error detecting and error correcting codes", Bell Syst. Tech. J. 29, pp 147-160. 